\title{
Rote Linien: Kontakt- und Informationsforum für SVV-Angehörige
}

\author{
"Line»
}

www.rotelinien.de

\section{Mein Weg zur Gründung einer SVV-Selbsthilfegruppe}

\section{Persönliche Konfrontation mit selbstverletzendem Verhalten} $(S V V)$

Im Alter von 13 Jahren hat meine Tochter, die jüngste von drei Geschwistern, in einer persönlichen Krisensituation angefangen, sich zu selbst zu verletzen. Als Mutter habe ich sehr schnell gemerkt, dass SVV ein Tabu-Thema ist und ein offener, unkomplizierter Umgang damit genauso wenig möglich ist wie kurzfristige Unterstützung. Vom kontaktierten Hausarzt erhoffte ich mir Hilfe, aber er war ganz klar überfordert. Er kannte sich fachlich nicht aus, und auch die Wege zu einer Therapie waren ihm unklar. Niedergelassene Psychotherapeuten hatten monatelange Wartezeiten und waren wider Erwarten auch nicht unbedingt mit Autoaggression vertraut, von daher genauso wenig hilfreich. Eine Beratungsstelle zeigte sich dann glücklicherweise kompetent im Umgang mit SVV, konnte uns kurzfristig einen Termin für ein erstes Elterngespräch anbieten und gab unserem Problem einen Namen: SVV - selbstverletzendes Verhalten. Sie versorgte uns Eltern mit ersten, fachlichen Informationen und bot unserer Tochter umgehend eine ambulante Therapie an.

\section{Selbstverletzendes Verhalten im Internet}

Um mehr über das Thema zu erfahren, las ich Bücher über SVV und suchte nach Informationen im Internet. Hier konnte ich anfangs vor allem über die amerikanische Seite «Secret Shame» [www.palace.net/ llama/psych/injury.html] Erfahrungen austauschen. Offen berichteten mir Betroffene per Mail über ihre Lebenssituation, was sie taten und wie sie sich fühlten. Der Austausch war für mich zum Verständnis wichtig, da unsere Tochter nicht mit uns über ihre Gefühle, Ängste und Probleme reden konnte, ich sie aber verstehen wollte. Es entstanden einige wenige, aber sehr wohltuende Kontakte zu anderen Müttern. Letztlich war der Austausch aber längerfristig zu kompliziert und zeitaufwendig, weil er auf Englisch stattfinden musste. Im deutschsprachigen Raum Eltern zu finden, gestaltete sich schwierig. Die wenigen, die im Internet surften, gingen mit ihren Beiträgen in den SVV-Foren unter. Die Stimmung war auch nicht immer so, dass ich mich als Mutter auf Betroffenen-Seiten willkommen fühlte. Da erschien es logisch, eine Plattform speziell für SVV-Angehörige, also für Familienmitglieder, Lebenspartner und enge Freunde, einzurichten.

\section{Erstellung einer Internetpräsenz für SVV-Angehörige}

Im Februar 2002 stellte ich die Homepage «Rote Linien Kontakt- und Informationsforum für SVV-Angehörige» [www.rotelinien.de] online. Die Seite wird von Angehörigen, aber auch von aktiven und ehemaligen Selbstverletzern genutzt. Auf der Homepage habe ich all die Informationen zusammengestellt, die ich mir bei der Konfrontation mit SVV gewünscht hätte. Neben der Bereitstellung von allgemein verständlichen Informationen über SVV, Lebensgeschichten, Hilfe-Adressen usw. sind mir vor allem der Erfahrungsaustausch und die gegenseitige Unterstützung wichtig. Diese können via Forum, Chat, E-Mail und Messengersystemen (beispielsweise ICQ) erfolgen. Gerade der Austausch von Angehörigen und Betroffenen wird als sehr hilfreich empfunden, weil er dazu dient, die jeweils andere Seite mit ihren speziellen Sorgen und Nöten verstehen zu lernen und damit besser im eigenen Umfeld zurecht zu kommen.

\section{«Selbsthilfegruppe» im Internet}

Über die Rote-Linien-Homepage hat sich vor etwa einem Jahr eine feste Gruppe von zehn Betroffenen und Angehörigen gebildet. Diese nutzt zur Kommunikation untereinander ein nicht öffentliches Forum und einen separaten Chatraum. Persönliche Treffen der Gruppe haben wegen der großen Entfernung der Wohnorte erst dreimal stattgefunden. Anfragen

\begin{tabular}{ll}
\hline KARGER & @ 2004 S. Karger GmbH, Freiburg \\
$\begin{array}{l}\text { Fax +497614520714 } \\
\begin{array}{l}\text { E-mail Information@Karger.de } \\
\text { www.karger.com }\end{array}\end{array}$ & $\begin{array}{l}\text { Accessible online at: } \\
\text { www.karger.com/dps }\end{array}$
\end{tabular}

\section{KARGER}

www.karger.com
Webmaster der Rote-Linien-Homepage

E-mail selbsthilfe@rotelinien.de

www.rotelinien.de 
bei zwei verschiedenen Krankenkassen ergaben, dass es bislang keine Kriterien zur Anerkennung und Förderung von überwiegend per Internet agierenden Gruppen gibt. Ein Zustand, der nicht zeitgemäß erscheint.

\section{Gründung einer lokalen Selbsthilfegruppe}

Nach den durchweg positiven Erfahrungen der aus Angehörigen und Betroffenen bestehenden Internet-Gruppe habe ich die Gründung einer lokalen Selbsthilfegruppe mit der gleichen Konstellation angestrebt.

Es waren mehrere Gespräche mit der Selbsthilfekontaktstelle notwendig, um die Gründung vorzubereiten. Um im Landkreis auf die Thematik SVV und auf die zukünftige Gruppe aufmerksam zu machen, habe ich Handzettel mit leicht verständlichen Informationen über SVV sowie einer Kontaktadresse entworfen, die kreisweit an bestimmte Institutionen (unter anderem Jugendzentren, Beratungsstellen, Ämter, Arztpraxen) verschickt wurden. Im Weiteren habe ich in $\mathrm{Zu}$ sammenarbeit mit einer Beratungsstelle und der Selbsthilfekontaktstelle eine Informationsveranstaltung über SVV organisiert. Entsprechende Pressemitteilungen haben auf die Informationsveranstaltung und die zu gründende Selbsthilfegruppe aufmerksam gemacht.

Die Selbsthilfegruppe hat sich bisher dreimal getroffen und steckt somit noch in der Anfangsphase. Die Anzahl der Teilnehmer und die Offenheit, mit der über die Probleme gesprochen werden kann, gibt aber Anlass zur Hoffnung, dass diese Gruppe Bestand haben wird. Sie wäre damit eine von bundesweit fünf existierenden SVV-Selbsthilfegruppen. Fünf Gruppen bei schätzungsweise 800000 Betroffenen und einer entsprechend größeren Anzahl an Angehörigen ...

\section{Zusatzinformationen}

Lokale SVV-Selbsthilfegruppen gibt es in:

Berlin (SHG für betroffene Frauen; Kontakt über Selbsthilfe Neukölln)

www.selbsthilfe-neukoelln.de

Hannover (SHG für betroffene Frauen; Kontakt über KIBIS/KISS)

www.kibis-kiss.de/pages/fr/frameset_gruppen.htm

Hanau (SHG für Angehörige; Kontakt über SEKOS) www.selbsthilfekontakstelle.de

Lüneburg (SHG für betroffene Frauen; Kontakt über KIBIS des Paritätischen)

www.kibis-lueneburg.de

Lünen (SHG für Angehörige und Betroffene; Kontakt über KISS)

www.selbsthilfenetz.de/e10/e102/index_ger.html?instanz_id= 46 